\title{
Human papillomavirus knowledge and intent to vaccinate among young college age males
}

\author{
Eric Fenkl, Irina Hughes, Sandra Gracia Jones \\ Nicole Wertheim College of Nursing and Health Sciences, Florida International University, Miami, Florida, USA
}

Received: May 26, 2016

DOI: $10.5430 /$ jer.v2n2p108
Accepted: June 26, 2016

Online Published: June 30, 2016

URL: http://dx.doi.org/10.5430/jer.v2n2p108

\begin{abstract}
Human papillomavirus (HPV) is the most common sexually transmitted infection (STI) in the United States and globally. The virus has the ability to be transmitted through sexual contact (vaginal, anal, and/or oral sex) and/or genital-to-genital (skin) contact. HPV types $6,11,16,18,31,33$, and 45 are considered high risk factors for the development of anal, oral, and penile cancers among the male population. Unfortunately, there are no widely used screening tests for HPV-related anal, oral or penile cancers. This has allowed the virus to remain undetected and, as a result, the rate of male mortality to increase. Although the HPV vaccine has been recommended for both females and males 9 through 26 years of age, more females than males have received the vaccination. The purpose of this study was to assess knowledge of HPV, HPV-related disease, and intent to vaccinate among young college age males. Following IRB approval, a sample of 131 male students completed a self-administered, 54-question, paper-and-pencil questionnaire. The findings of this study indicated that a majority of participants had a general knowledge about HPV virus, many of the participants were unaware that HPV could present with no outward associated symptomology, that bleeding or bloody discharge could be a symptom of anal cancer, and that there is no cure for HPV infection. $60 \%$ were unaware of the availability of the HPV vaccine for male, greatly limiting the intent to potentially vaccinate. Healthcare providers should provide young males with information related to HPV infection, HPV-related disease, and vaccination.
\end{abstract}

Key Words: Human papillomavirus, Males, Knowledge, Vaccination

\section{INTRODUCTION}

Human papillomavirus (HPV) is the most common sexually transmitted infection (STI) in the United States ${ }^{[1]}$ and globally. ${ }^{[2]}$ More than 100 types of HPV virus are known and approximately 40 different types are responsible for HPVrelated diseases affecting both women and men. The virus has the ability to be transmitted through sexual contact (vaginal, anal, and/or oral sex) and/or genital-to-genital (skin) contact. HPV types $6,11,16,18,31,33$, and 45 are considered high risk factors for the development of anal, oral, and penile cancers among the male population. ${ }^{[3]}$ The HPV virus presents with no outward signs or symptoms of infection, therefore it is challenging to educate young adults about a disease they cannot visualize or that does not produce symptoms for years. ${ }^{[4]}$ Gerend and Barley ${ }^{[5]}$ reported a major deficit in HPV knowledge among males aged 18-26 years, attending a Florida public university in 2009. Similarly, Stock, Peterson, Houlihan, and Walsh, ${ }^{[6]}$ reported measures of oral sex and HPV knowledge were higher for females in comparison to males. Unfortunately, there are no widely used screening

\footnotetext{
${ }^{*}$ Correspondence: Eric Fenkl; Email: efenkl@ fiu.edu; Address: Nicole Wertheim College of Nursing and Health Sciencesn, Florida International University, Miami, Florida, USA.
} 
tests for HPV-related anal, oral or penile cancers. ${ }^{[7]}$ This has allowed the virus to remain undetected and, as a result, the rate of male mortality to increase. The goal of Healthy People 2020 is to substantially reduce the number of HPVrelated infections in the United States. ${ }^{[8]}$ One of the steps in this approach is to protect against specific types of HPV through vaccination. HPV vaccines provide a developing immune response, producing antibodies after the first dose. Human papillomavirus Quadrivalent Vaccine (Gardasil $($ ) has been approved and recommended for vaccination of both females and males 9 through 26 years of age. Seroconversion after receiving the third dose of the quadrivalent vaccine has previously demonstrated an efficacy of $97.5 \%{ }^{[9]}$. While vaccination rates have increased among females, males continue to lag. ${ }^{[10]}$ One reason for this is that primary care providers are not consistently recommending vaccination for adolescent boys. For young men, 18 years and older, vaccination against HPV has been perceived largely as a female specific or genital specific concern. Even in male populations at greatest risk, such as HIV-infected and non-infected men who have sex with men (MSM), previous findings have indicated HPV knowledge deficits. ${ }^{[1,12]}$ The purpose of this study was to assess knowledge of HPV, HPV-related disease, and intent to vaccinate among young college age males. No previous studies to assess such knowledge in this sample and location were identified.

\section{Methodology}

Following Institutional Review Board (IRB) approval, a sample of 131 male students completed a self-administered, 54question, paper-and-pencil questionnaire. The questionnaire was modified from one previously used by Schaefer-Ziemer and Hoffman. ${ }^{[13]}$ Study participants were advised both orally and with a written informational letter of the purpose of the research. Participants were informed that they could opt out of participating in the study at any point for any reason and could choose not to respond to any questions that they did not wish to answer. Inclusion criteria were being a male between the ages of 18 and 26 years and enrolled as a student at the university. The data collection site for this study was the student clinic at a large, urban, minority-serving University in South Florida. No investigator conflicts of interest were identified.

\section{RESUlts}

The mean age of participants $(\mathrm{N}=131)$ was 22 years. The majority of participants were undergraduate students, 85 $(65 \%)$ and $46(35 \%)$ were graduate students. In terms of race, more than half of the students $100(77 \%)$ identified as White, $20(15 \%)$ as African American, $9(7 \%)$ Asian and 2 (1\%) Native American. Regarding ethnicity, 54\% identified as Hispanic/Latino. In terms of sexual orientation, the majority identified as heterosexual (84\%), $8 \%$ as gay, $4 \%$ as bisexual and $4 \%$ did not specify a sexual orientation.

Table 1. Summary of Findings $(n=131)$

\begin{tabular}{|c|c|c|}
\hline Items & $\mathbf{n}$ & $\%$ \\
\hline The participants recognized risk factors of contracting HPV through multiple sex partners. & 99 & $76 \%$ \\
\hline The participants recognized risk factors of contracting HPV through partners who had multiple sex partners. & 87 & $67 \%$ \\
\hline The participants recognized risk factors of contracting HPV through failure to use condoms. & 88 & $67 \%$ \\
\hline The participants recognized risk factors of contracting HPV through early sexual activity (38\%). & 49 & $38 \%$ \\
\hline The participants were aware that transmission of HPV infection can occur through genital skin-to-skin contact. & 67 & $51 \%$ \\
\hline The participants were aware that transmission of HPV infection can occur through contact with bodily fluid. & 63 & $48 \%$ \\
\hline The participants were aware that transmission of HPV infection can occur through oral sex. & 61 & $47 \%$ \\
\hline The participants were aware that transmission of HPV infection can occur through anal sex. & 63 & $48 \%$ \\
\hline The participants were aware that HPV could present with no outward associated symptomology. & 20 & $15 \%$ \\
\hline The participants were aware that bleeding or bloody discharge could be a symptom of anal cancer. & 33 & $25 \%$ \\
\hline The participants were aware that there is no cure for HPV infection. & 39 & $30 \%$ \\
\hline The participants were aware that of the availability of the HPV vaccine for males. & 52 & $40 \%$ \\
\hline
\end{tabular}

The findings of this study (see Table 1) indicated that a majority of participants had a general knowledge about HPV virus. The participants correctly recognized risk factors of contracting HPV through multiple sex partners $(76 \%)$ or partners who had multiple sex partners (67\%), failure to use condoms $(67 \%)$, and early sexual activity (38\%). They showed awareness of the transmission of HPV infection through genital skin-to-skin contact (51\%), contact with bodily fluid (48\%), and oral $(47 \%)$ and anal sex $(48 \%)$. Additionally, $85 \%$ were unaware that HPV could present with no outward associated symptomology, $75 \%$ were unaware that bleeding or bloody discharge could be a symptom of anal cancer, and $70 \%$ were unaware that there is no cure for HPV infection. Finally, 79 $(60 \%)$ of the participants reported not being aware of the 
availability of the HPV vaccine for males, greatly limiting the intent to potentially vaccinate.

\section{Limitations}

This study used a convenience sample which may limit the generalization of the findings to other young collge age malesen within South Florida and other areas of the U.S. study results may be more descriptive of this particular group of young men rather than men in the general population.

\section{Discussion}

Despite being able to identify the correct factors of transmission HPV infection, the number of participants who identified oral and anal sex as safe was alarming. It was equally disquieting to see that the majority of participants were not aware that there was no outward sympotomology associated with HPV, thus reinforcing the need for vigilant screening, particularly among those individuals at greater risk for HPV related infection and disease. The number of males in this study who reported not being aware of a vaccine for HPV further demonstrates the need for more HPV prevention outreach. Healthcare providers and other public health related professionals and community advocates should take advantage of every encounter with young males to provide them with information related to HPV infection and HPV-related disease including the benefits of vaccination. For those young males potentially at high risk, screening measures such as anal pap smears may be warranted. Additionally, healthcare providers should screen for vaccination history and inform young males about availability and benefits of HPV vaccination. Only through such measures will healthcare providers concerned with the health of men be able to promote the goal of Healthy People 2020 to substantially reduce the number of HPV-related infections in the United States. ${ }^{[5]}$

\section{REFERENCES}

[1] National Cancer Institute. HPV and cancer. 2014. Available from: http://www.cancer.gov/cancertopics/factsheet/ Risk/HPV

[2] World Health Organization (WHO) Immunizations, Vaccines, and Biologicals: Human Papillomavirus. Available from: http://www . who.int/immunization/topics/hpv/en/

[3] Dunne EF, Nielsen CM, Stone, KM, et al. Prevalence of HPV Infection among Men: A Systematic Review of the Literature. J of Infectious Diseases. 2006; 194: 1044-57. PMid:16991079 http://dx.doi.org/10.1086/507432

[4] Centers for Disease Control and Prevention. Sexual transmitted Infection: Genital HPV Infection-fact sheet. 2013. Available from: http://www.cdc.gov/std/hpv/stdfact-hpv.htm.

[5] Gerend MA, Barley J. Human papillomavirus vaccine acceptability among young adult men. Sex Transm Disease. 2009; 36(1): 58-62. http://dx.doi.org/10.1097/OLQ.0b013e31818606f c

[6] Stock ML, Peterson LM, Houlihan AE, et al. Influence of oral sex and oral cancer information on young adults' oral sexual-risk cognitions and likelihood of HPV vaccination. Journal of Sex Research. 2013; 50(1): 95-102. http://dx.doi.org/10.1080/00224499. 2011.642904
[7] Centers for Disease Control and Prevention. HPV and Men CDC Fact Sheet. Available from: http://www.cdc.gov/std/hpv/hp vandmen-fact-sheet-february-2012.pdf

[8] U.S. Department of Health \& Human Services. Human papilloma virus. 2000. Available from: http://www.hhs.gov/

[9] Giuliano AR, Palefsky JM, Goldstone S, et al. Efficacy of Quadrivalent HPV Vaccine against HPV Infection and Disease in Males. N Engl J Med. 2011; 364: 401-411. http: //dx.doi.org/10. 1056 /NEJMoa0909537

[10] Centers for Disease Control and Prevention. Vaccines and immunizations: Human Papillomavirus. 2015. Available from: http: //www.cdc.gov/vaccines/pubs/pinkbook/hpv.html.

[11] Fenkl EA, Schochet E, Jones SG, et al. Evaluation of an HPV/Anal Cancer Screening Awareness Program for HIV-infected Men Who Have Sex With Men. Journal of the Association of Nurses in AIDS Care. 2015; 26(4): 492-497. PMid:25682353 http://dx. doi.org /10.1016/j.jana.2015.01.003

[12] Fenkl EA, Jones SG, Schochet E, et al. HPV and Anal Cancer Knowledge among HIV-infected and non-infected Men who have Sex with Men. LGBT Health. 2016; 3(1): 43-8.

[13] Schaefer-Ziemer K, Hoffman MA. Beliefs and attitudes regarding HPV vaccination among college-age women. Journal of Health Psychology. 2013; 18(10): 1360-1370. PMid:23188917 http://dx.d oi.org/10.1177/1359105312462432 